\title{
MOEMS-based spectro-imagers for Earth and Universe Observation
}

Mathieu Vachey, Frédéric Zamkotsian, Hervé Benard, Arnaud Liotard, Nicolas Tetaz, et al.

Mathieu Vachey, Frédéric Zamkotsian, Hervé Benard, Arnaud Liotard, Nicolas Tetaz, Vincent Costes, "MOEMS-based spectro-imagers for Earth and Universe Observation," Proc. SPIE 11852, International Conference on Space Optics - ICSO 2020, 118523N (11 June 2021); doi: 10.1117/12.2599614

SPIE Event: International Conference on Space Optics - ICSO 2021, 2021, Online Only 


\section{International Conference on Space Optics-ICSO 2020}

Virtual Conference

30 March-2 April 2021

Edited by Bruno Cugny, Zoran Sodnik, and Nikos Karafolas
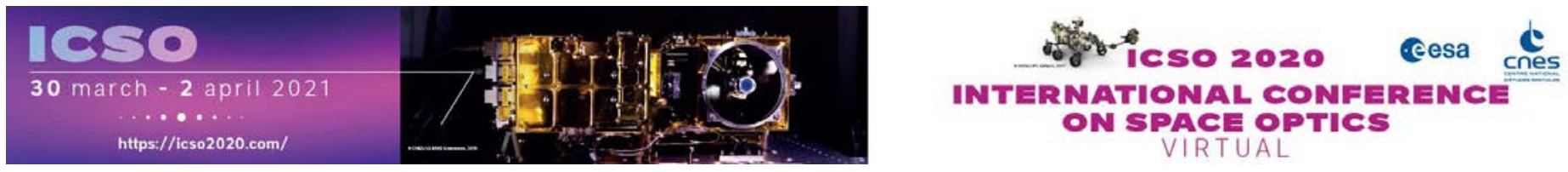

\section{MOEMS-based spectro-imagers for Earth and Universe Observation}

\section{Cesa issopocesalings lecnes}




\title{
MOEMS-based spectro-imagers for Earth and Universe Observation
}

\author{
Mathieu Vachey ${ }^{\mathrm{a}}$, Frédéric Zamkotsian ${ }^{\mathrm{a}}$ \\ Hervé Benard ${ }^{\mathrm{b}}$, Arnaud Liotard ${ }^{\mathrm{b}}$, Nicolas Tetaz ${ }^{\mathrm{b}}$, Vincent Costes $^{\mathrm{c}}$ \\ a Aix Marseille Univ, CNRS, CNES, LAM - Laboratoire d'Astrophysique de Marseille, \\ 38 rue Frédéric Joliot Curie, 13388 Marseille Cedex 13, France \\ ${ }^{\mathrm{b}}$ Thales Alenia Space, 100 Boulevard du Midi, BP 99, 06156 Cannes la Bocca Cedex, France \\ ${ }^{\mathrm{c}}$ CNES, 18 Avenue Edouard Belin, 31401 Toulouse Cedex 9, France \\ e-mail address: frederic.zamkotsian@lam.fr
}

\begin{abstract}
MOEMS-based instruments could be the next stepping stone in ground-based and space telescopes for Universe and Earth Observation. Targeted instruments are next generation multi-object spectrographs, as MOEMS can be used as reconfigurable slit masks at the MOS entrance, allowing increased instrument compacity and new observational modes such as SNR optimization. For Earth Observation, the use of MOEMS acting like programmable slit masks also allows a dynamic removal of the bright sources in the field of view, therefore enhancing the global SNR of the instrument.

We propose a MOEMS-based spectro-imager named BATMAN, using a Digital-Micromirror-Device (DMD) to split the light between its imaging and spectrograph arms. This instrument is intended to be installed at the TNG telescope in the Canary Islands in 2022. This concept has been extended for space Universe and Earth Observation.

A new MOEMS-based spectro-imager for Earth Observation has been designed, with constraints of wide 2D field-ofview $\left(3^{\circ} \times 1^{\circ}\right)$, image quality $(<2$ pixels $=11 \mu \mathrm{m})$ and compacity (Fig. 1$)$. The instrument is panchromatic with a medium spectral resolution between 1000 and 2000, fitting in a $80 \mathrm{~cm}$ x $80 \mathrm{~cm}$ x $40 \mathrm{~cm}$ box. A 3-mirror solution for both imaging and spectrograph arms has been designed, using only aspheric surfaces, allowing for easier alignment and tolerancing. The compact design contains a convex grating to disperse light. In order to optimize the spectrograph efficiency, this convex grating must be blazed at the right angle for maximizing the light in the first order of diffraction.

For Universe Observation, a new design for a DMD-based high-resolution spectro-imager has been done. For the spectrograph, the large $370-950 \mathrm{~nm}$ wavelength range is divided into 4 channels for a spectral resolution of 15000 , all with an image quality of under two pixels. Moreover, the 3-mirror design of the instrument allows for a high throughput in comparison to classical systems which use catadioptric optics.

These results show the great interest of MOEMS-based spectro-imagers for space Earth and Universe Observation.
\end{abstract}

Keywords: Spectro-imager, MOEMS, Earth observation, Universe observation, high spatial resolution, high spectral resolution.

\section{INTRODUCTION}

For ground-based and space-borne telescopes, spectro-imagers are essential instruments: simultaneous imagery and spectroscopy increases both instrument and observation efficiency. To fully exploit Earth surface observation as well as astronomical objects observations, spectro-imagers must be optimized in terms of spatial and spectral resolution over the whole field of view (FOV).

While many satellites already observe the Earth at high spatial resolution, the need for hyperspectral imaging in Earth observation increases. Medium spatial and spectral resolutions along a large FOV and Multi-Object Spectroscopy (MOS) allow for fast mapping of the Earth surface such as agricultural lands, forests or oceans. With goals of improving food security, agriculture and land characterization, such instruments are often considered for incoming space-borne missions. A spectro-imager for Earth observation adapted for this type of space mission is to be described in this paper. 
In Universe Observation, science cases are ranging from remote galaxies to our galaxy and star physics: examples such as the observation of young star-forming clusters ${ }^{1}$, XUV galaxies with high star formation rate ${ }^{2}$ or turbulence at intermediate redshift ${ }^{3}$ require both high spectral and spatial resolution over a large FOV. For these astronomical observations, fiber bundles ${ }^{4,5}$ are usually considered to select the objects in the FOV, limiting the global instrument transmission and photometry.

For standard observation of astronomical objects and the large FOV provided by the best telescopes, Multi-Object Spectroscopy (MOS) also features many advantages: by enabling the recording of hundreds of spectra over a single observation run, the efficiency of the scientific return obtained by MOS is essential for every spectrograph, and most major telescopes are already equipped with it. MOS is even more efficient when combined with slit spectroscopy, ensuring for high precision spectra, optimization of the Signal-to-Noise Ratio (SNR) and removal of background light in the spectrograph.

Micro-Opto-Electro-Mechanical-Systems (MOEMS) are a promising solution for slit pattern generation in MOS. They appear as micro-mirror arrays (MMA) ${ }^{6,7}$ or micro-shutter arrays (MSA) ${ }^{8}$ and, when placed in the focal plane of the telescope, act as programmable slit masks over the whole FOV. By individually controlling each micro-mirror, slit patterns can be generated in real time and different slit widths allow variable spectral resolutions and spatial sampling. Two candidates are considered in this work: a commercially available device, the Digital Micromirror Device (DMD) from Texas instrument and a custom-designed MMA developed by LAM together with EPFL and CSEM (Switzerland). The DMD features more than 2 million micro-mirrors $(2048 \times 1080)$ for a total size of $28 \mathrm{~mm} \times 14 \mathrm{~mm}$. Each mirror is $13.5 \mu \mathrm{m}^{2}$ and can be individually switched between two positions $+12^{\circ}$ (so called ON) and an $-12^{\circ}$ (so called OFF). DMDs have been tested in spatial environment, showing no showstopper to operate at low temperatures, in vacuum and under radiations conditions 9 . The European development of MEMS-based programmable reflective slit masks from the jointed effort of LAM, EPFL and CSEM is another candidate. This MOEMS device consists in a micro-mirror array, each single crystalline silicon micro-mirror being $100 \mu \mathrm{m}$ × $200 \mu \mathrm{m}$ wide and providing a precise tilt angle of $0^{\circ}$ or $-20^{\circ}$ due to the electrostatic force. Micro-mirror arrays with a $-20^{\circ}$ angle have already been successfully realized and tested in cryogenic environment. ${ }^{10}$

A MOEMS-based spectro-imager concept called BATMAN ${ }^{11}$ has been studied by a French-Italian consortium and is to be mounted on the TNG telescope (3.58 m primary) in the Canaria Islands. The DMD is placed in the focal plane of the telescope, where micro-mirrors act as programmable slit masks for the spectrograph. From the DMD, the light from the selected objects is sent towards the spectrograph while the remaining light is sent towards the imager, both using Offner relays to re-image on their respective CCD.

From BATMAN development, two new MOEMS-based spectro-imager designs for Earth and Universe observation are presented in this paper. The first design will overview the proposed spectro-imager for Earth observation and the second design will discuss the spectro-imager design for Universe observation.

\section{SPECTRO-IMAGER FOR EARTH OBSERVATION}

For Earth Observation, the mission type is oriented towards the observation and characterization of Earth surfaces such as forests and agricultural lands with in mind the goal of improving food security, agriculture and land characterization. The observation of large Earth spatial elements requires a spatial resolution better than $30 \mathrm{~m} / 2$ detector pixels; instruments such as CHIME ${ }^{12}$, a hyperspectral imager for the environment scheduled for the mid-2020, or ELOIS ${ }^{13}$, a free-form spectrometer for Earth observation, are looking for comparable spatial resolutions. The spatial resolution for our Earth observation spectro-imager must be then lower than $30 \mathrm{~m} / 2$ detector pixels.

A wavelength range of $400 \mathrm{~nm}-800 \mathrm{~nm}$ is selected; principal examples of observation within this wavelength range are crop characteristics such as chlorophyll content or photosynthetically active vegetation. Resolving these science cases requires a medium spectral resolution higher than 1000. To ensure proper observations of the Earth's surface, the spectral resolution of the spectrograph is then set as greater than 1000 . 
The radiometric requirement is key for a space-borne instrument and is related to the entrance pupil diameter (EPD) of the entry telescope i.e. diameter of the telescope's primary mirror. Typical instruments for hyperspectral imaging such as GOMOS $^{14}$ have entrance pupil diameters between $200 \mathrm{~mm}$ and $300 \mathrm{~mm}$ to ensure instrument photometry. The EPD for the MOEMS-based spectro-imager is chosen with CNES as equal to $200 \mathrm{~mm}$, insuring for a small entry telescope while still meeting the instrument radiometric requirement.

For space instruments looking at the Earth, their FOV are often chosen as linear and operating in push-broom mode. Instruments like Pléiades ${ }^{15}$ or Spot $^{16}$ take advantage of a very high spatial resolution by looking at a linear FOV with values respectively equal to $1.65^{\circ}$ and $8.4^{\circ}$. For imagery, a linear FOV in push-broom mode results in fast mapping of the Earth, however, for spectroscopy, the addition of a secondary dimension to the FOV allows to fully exploit the capabilities of the MOEMS device: by controlling each micro-mirror individually, slit spectroscopy, MOS and IFU are available over the whole FOV. Moreover, fast switching of the micro-mirrors allows for quasi-simultaneous imagery and spectroscopy over the FOV at any given time. Following this idea of increased spectroscopic abilities, the FOV is therefore chosen as rectangular and equal to $3^{\circ} \mathrm{x} 1^{\circ}$.

\subsection{Design overview}

A new MOEMS-based spectro-imager design for Earth observation has been designed following CNES requirements (Fig. 1). A typical $3^{\circ} \times 1^{\circ} \mathrm{FOV}$ is imaged at F/5 on the MOEMS plane by the entry telescope for a $63 \mathrm{~mm} \times 21 \mathrm{~mm}$ intermediate focal plane. The DMD features only $28 \mathrm{~mm} \times 14 \mathrm{~mm}$ and is no longer considered for this type of space mission; a custom MMA called MIRA $^{17}$ is selected as the MOEMS device to relay the light to both imager and spectrograph. The intermediate plane is composed of three MIRA arrays, each featuring 420 × 420 micromirrors for a total $1260 \times 420$ micromirrors on the array. When MIRA micro-mirrors are tilted on the ON position, the light from the selected objects is directed to the spectrograph, achieving a mean spectral resolution of 1,500 . When the micro-mirrors are tilted in the OFF position, the light is directed towards the imager. The spatial resolution of the instrument must be better than $30 \mathrm{~m} / 2$ detector pixels and a challenging spatial resolution of $9 \mathrm{~m} / 2$ detector pixels is reached over the $3^{\circ} \times 1^{\circ} \mathrm{FOV}$.

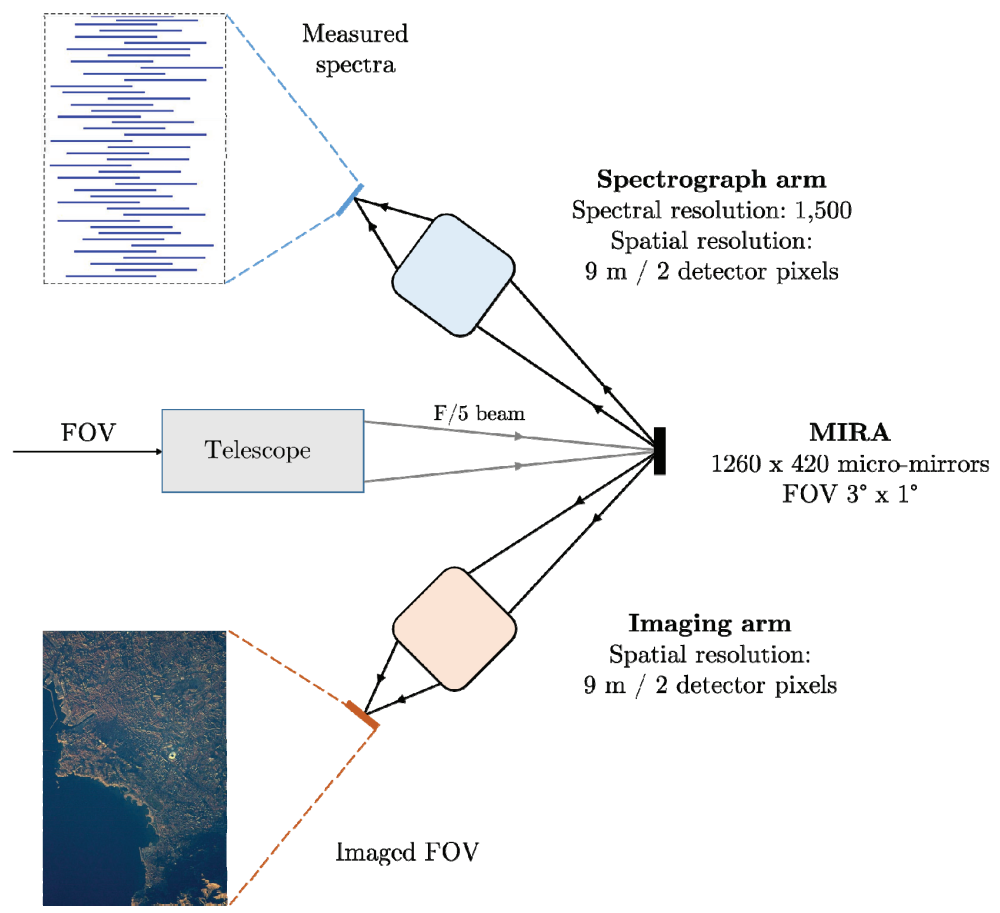

Fig. 1: Earth observation MOEMS-based spectro-imager concept: the FOV is a space view of the city of Marseille shining the entry telescope, incoming from the left and is imaged by the entry telescope on MIRA. The selected objects in the FOV are sent to the spectrograph arm (upwards), while the remaining light is sent to the imaging arm (downwards). 


\subsection{Instrument specifications}

The $3^{\circ} \times 1^{\circ} \mathrm{FOV}$ is projected on a $63 \mathrm{~mm} \times 21 \mathrm{~mm}$ intermediate focal plane at $\mathrm{F} / 5$. This focal plane features three [21 mm x $21 \mathrm{~mm}$ ] MIRA arrays lying next to each other in a 3:1 rectangle. The F/5 beam is imaged on $1260 \mathrm{x} 420$ micro-mirrors of $50 \mu \mathrm{m} \times 50 \mu \mathrm{m}$ size for a platescale of 0.14 arcmin per micro-mirror. The light incomes MIRA with a $10^{\circ}$ incidence and with the micro-mirror positions being $0^{\circ}$ and $-20^{\circ}$, the beam then outcomes with $-20^{\circ}$ and $+20^{\circ}$ angles. For this kind of space mission, the usually considered detectors are CMOS CMV400 from CMOSIS, with 5.5 $\mu \mathrm{m}$ pixels in a $2 \mathrm{k}$ x $2 \mathrm{k}$ array for a $11 \mathrm{~mm}$ x $11 \mathrm{~mm}$ surface. Three CMOS detectors are chosen for the imager, all lying next to each other for a 3:1 detector plane. A spatial resolution of $9 \mathrm{~m} / 2$ detector pixels is therefore achieved. As both imager and spectrograph have the same spatial resolution, a larger detector surface is needed for the spectrograph to be able to display the spectra from all FOV points. This detector array is therefore extended to $3 \times 2$ CMOS detectors for the spectrograph.

The instrument specifications are summed up in table 1.

\section{Instrument specifications}

\begin{tabular}{cc}
\hline Entrance pupil diameter & $200 \mathrm{~mm}$ \\
\hline Field of view & $3^{\circ} \times 1^{\circ}$ \\
\hline Wavelength range & $400-800 \mathrm{~nm}$ \\
\hline Beams on MIRA & Incoming light at $10^{\circ}$ incidence \\
Micro-mirror positions: $0^{\circ} /-20^{\circ}$
\end{tabular}

Table 1: Spectro-imager for earth observation instrument specifications

\subsection{Imager}

The entry telescope focuses the $3^{\circ} \times 1^{\circ} \mathrm{FOV}$ on a $63 \mathrm{~mm} \times 21 \mathrm{~mm}$ intermediate focal plane made of three [21 mm x $21 \mathrm{~mm}$ ] MIRA arrays (Fig. 2). The light is then reflected to the imager and focuses on a $33 \mathrm{~mm} \times 11 \mathrm{~mm}$ detector plane for a 0.52 magnification between MIRA and the detector plane.

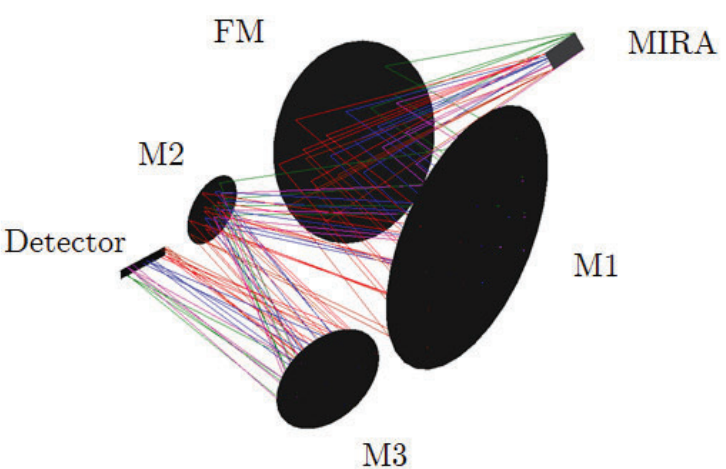

(a)

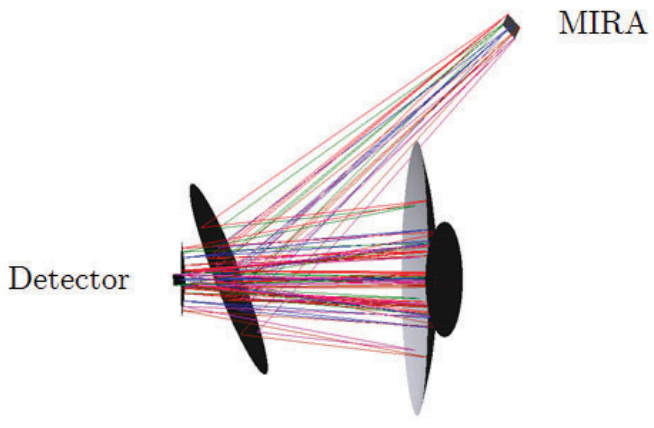

(b)

Fig. 2: Imaging arm of the Earth observation MOEMS-based spectro-imager

a) 3D imager layout, featuring MIRA, the FM, M1, M2, M3 and the detector plane; b) 3D imager layout along the imager plane. 
The imager is a concave-convex-concave TMA preceded by a fold mirror (FM), which breaks the $2 \mathrm{D}$ geometry of the instrument by shifting the M1 below the MIRA-FM beam. This mirror position also brings the imager and the spectrograph closer to each other for increased overall compacity. The layout of the imager starting at MIRA is shown along two different angles in Fig. 2. From top to bottom and along the light beam, it shows MIRA followed by the FM, M1, M2, M3 and the detector plane. The imager fits with MIRA in a $45 \mathrm{~cm} \mathrm{x} 40 \mathrm{~cm} \mathrm{x} 45 \mathrm{~cm}$ box.

Figure 3 shows three spot diagrams at the center vertical axis of the FOV and a contour color plot of the spot diagram values over the whole FOV projected on the detector plane.

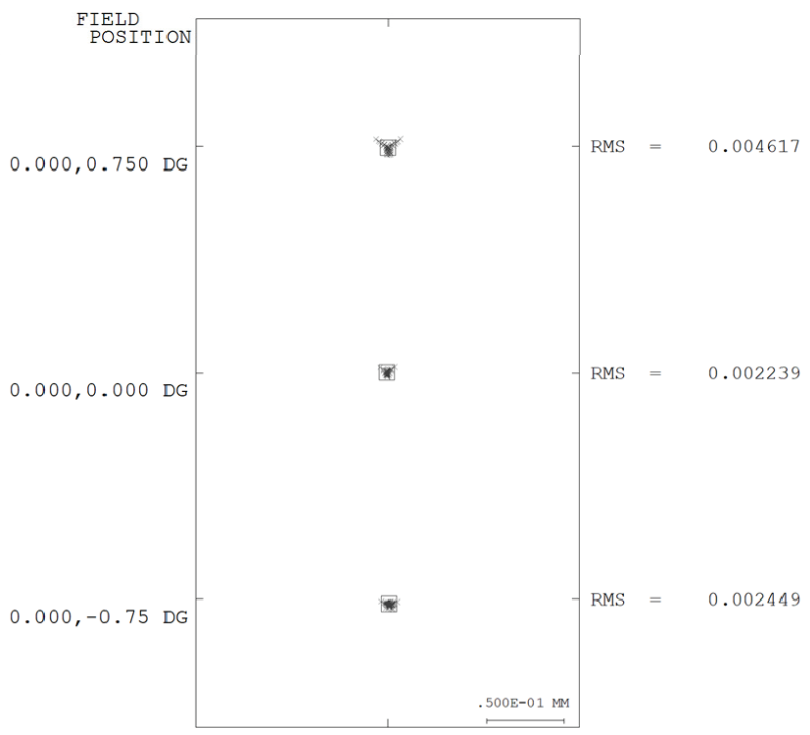

(a)

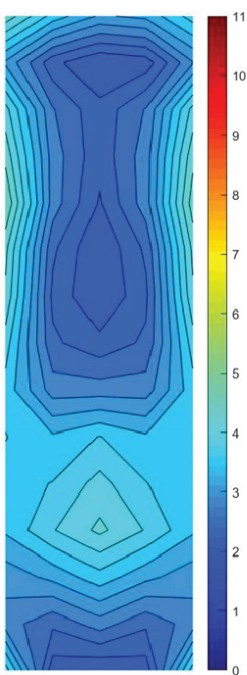

(b)

Fig. 3: Imager for Earth observation nominal image quality a) Spot diagrams $(\mu \mathrm{m})$ on the center vertical axis of the $3^{\circ} \mathrm{x} 1^{\circ} \mathrm{FOV}$ overlaid on top of a $5.5 \mu \mathrm{m}^{2}$ box representing the detector pixel; b) Spot diagram cartography for 75 points in the $3^{\circ} \times 1^{\circ} \mathrm{FOV}$.

The spot diagrams of Fig. 3a are close to the detector pixel size and Fig. 3b illustrates homogeneity of the spot diagrams over the FOV. All nominal spot diagrams are between $2.1 \mu \mathrm{m}$ and $4.6 \mu \mathrm{m}$ i.e. below one detector pixel. The mean image quality is equal to $3.2 \mu \mathrm{m}$ for a standard deviation of $0.1 \mu \mathrm{m}$, confirming the homogeneity of the imager shown in Fig. 3b.

The tolerancing procedure includes simulation of manufacturing errors on the mirrors, alignment errors and residual non-compensable errors. After 250 simulations for the imager, spot diagrams are increased by $3.5 \mu \mathrm{m}$ on average and reach a mean value after tolerancing of $6.8 \mu \mathrm{m}$ for the imager, close to 1.2 detector pixel. Few spot diagrams exceed the instrument specification of two detector pixels as 6220 out of the $6250 \mathrm{FOV}$ points remain under $11 \mu \mathrm{m}$, accounting for $99.5 \%$ of spot diagrams. These results confirm the possibility of alignment of the imager, meeting the last specification in terms of image quality after tolerancing.

\subsection{Spectrograph}

For the Earth observation spectrograph, the main specification is a spectral resolution higher than 1000 for the $400 \mathrm{~nm}-800 \mathrm{~nm}$ wavelength range. The same spatial resolution than for the imager is kept for the spectrograph: $9 \mathrm{~m} / 2$ detector pixels. The spectrograph also has the same entry object as the imager i.e. the intermediate focal plane of the $63 \mathrm{~mm} \times 21 \mathrm{~mm}$ MIRA plane. The spectrograph design is very similar to the imager, featuring three aspheric mirrors and a FM. The FM is placed in the same configuration as for the imager and minimizes the overall instrument volume. The second surface consists of an aspheric grating. All aspheric surfaces have maximum local slopes $<16 \mathrm{mrad}$, and then could be manufactured with current technologies. The layout of the spectrograph starting at MIRA is shown along two different angles in Fig. 4. 


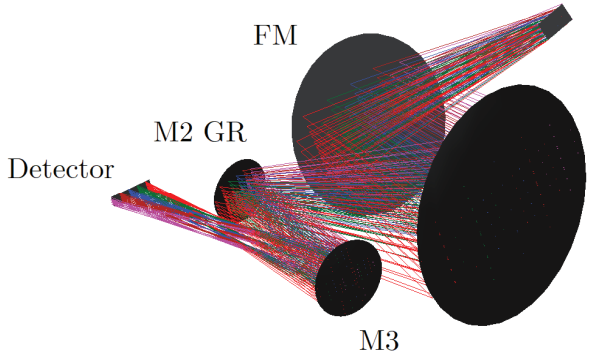

(a)

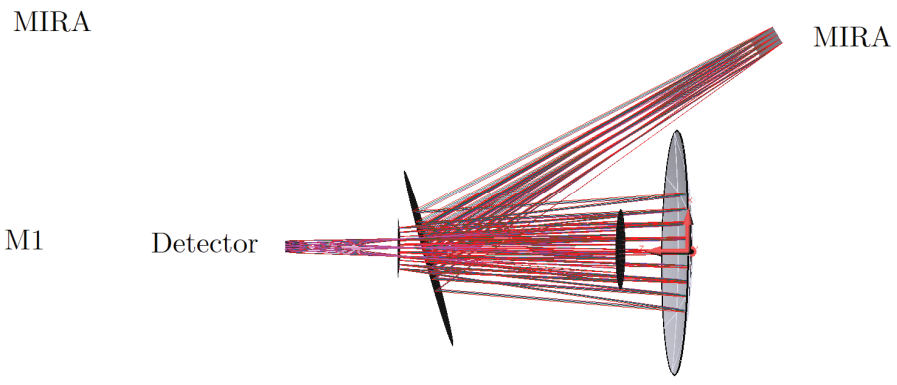

(b)

Fig. 4: Spectrograph arm of the Earth observation MOEMS-based spectro-imager a) 3D spectrograph layout, featuring MIRA, the FM, M1, M2 (grating), M3 and the detector plane; b) 3D spectrograph layout along the spectrograph plane.

A spot diagram cartography over the $3^{\circ} \times 1^{\circ} \mathrm{FOV}$ for the extreme and center wavelengths of the wavelength range is shown in Fig. 5. A maximum of $11 \mu \mathrm{m}$ is set for the colorbar as it is equivalent to two detector pixels.
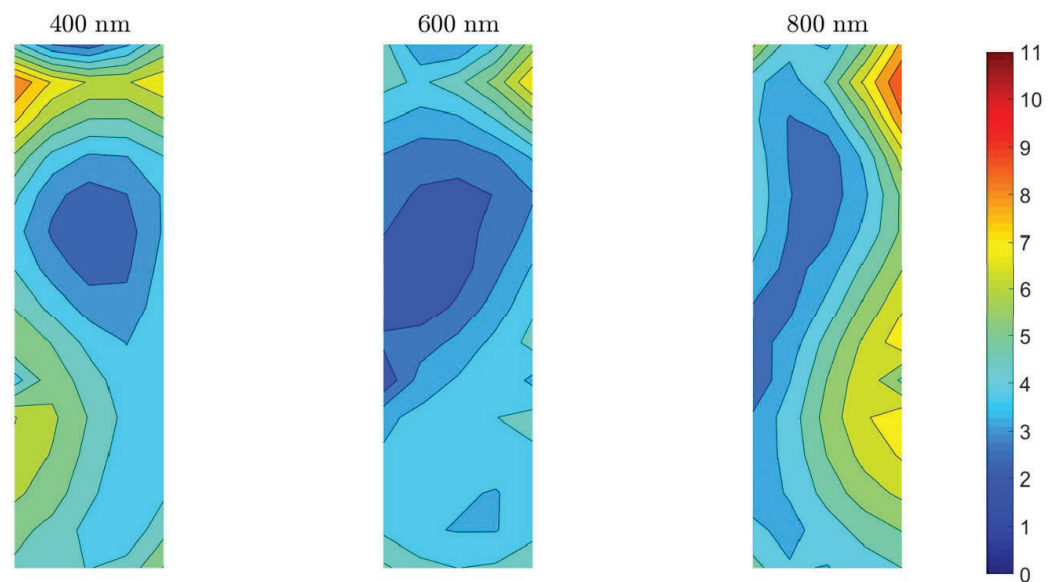

$\mu \mathrm{m}$

Fig. 5: Spectrograph for Earth observation nominal spot diagram cartography $(\mu \mathrm{m})$ for 225 points in the $3^{\circ} \times 1^{\circ} \mathrm{FOV}$ at three single wavelengths: $400 \mathrm{~nm}, 600 \mathrm{~nm}, 800 \mathrm{~nm}$.

The best spot diagram occurs at $600 \mathrm{~nm}$ and is equal to $2.0 \mu \mathrm{m}$ while the worst spot diagram appears at $800 \mathrm{~nm}$ for a value of $8.1 \mu \mathrm{m}$. For the center wavelength, no spot diagram exceeds $7.2 \mu \mathrm{m}$. The mean difference in spot diagram between center and extreme wavelengths is equal to $1.0 \mu \mathrm{m}$, demonstrating the spectrograph's homogeneity in terms of image quality.

The tolerancing process takes into account manufacturing errors on the mirrors, alignment errors and non-compensable errors. It is is applied to the spectrograph for $25 \mathrm{FOV}$ points at extreme and center wavelengths (i.e. 75 points per spectrograph) for 250 systems, which accounts for a total of 18,750 FOV points. After simulation, $99.2 \%$ of the spot diagrams are below $11 \mu \mathrm{m}$. The image quality of the spectrograph after tolerancing is confirmed to meet instrument specifications.

Spectra on the detector plane are shown in Fig. 6. Figure 6a features in red five spectra at the center and four edges of the FOV; their center and extreme wavelengths are highlighted in black. All five extreme spectra tightly fit in the detector array, their position are symmetric in both $\mathrm{X}$ and $\mathrm{Y}$ directions and their length are $11 \mathrm{~mm}$. Figure $6 \mathrm{~b}$ shows multiple spectra imaged at the same time on the detector array, illustrating the capabilities of MOEMS-based instruments to combine MOS and IFU over the whole $3^{\circ} \times 1^{\circ} \mathrm{FOV}$. 


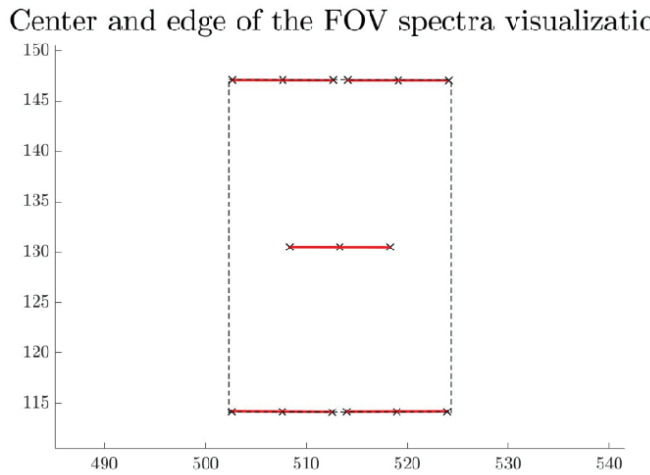

(a)

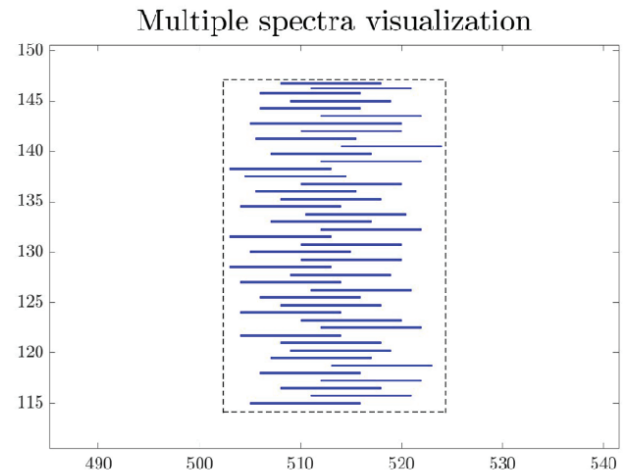

(b)

Fig. 6: Earth observation spectrograph detector plane view a) Spectra visualization for the center and edges of the FOV, overlaid on top of the detectors in dashed lines; b) Multiple spectra visualization overlaid on top of the detectors in dashed lines.

The spectral resolution of the spectrograph is plotted in table 2 .

\begin{tabular}{|c|c|c|c|c|}
\cline { 3 - 4 } & & B and 1 & B and width (nm) \\
\hline W avelength (nm) & 400 & 600 & 800 & 400 \\
\hline Spectral resolution & 1000 & 1500 & 2000 & \\
\hline
\end{tabular}

Table 2: Earth observation spectrograph spectral resolution over the $400-800 \mathrm{~nm}$ wavelength range

The starting spectral resolution is equal to 1000 at $400 \mathrm{~nm}$ and increases to 1500 at center wavelength. A maximum spectral resolution of 2000 is reached at $800 \mathrm{~nm}$. The average spectral resolution of the spectrograph is equal to 1500 , higher than the instrument specification.

\subsection{Spectro-imager}

An assembly of the two designs on each side of MIRA leads to the final layout of the spectro-imager and the result is presented in Fig. 7, shows both spectrograph (left) and imager (right) on each side of the MIRA plane. The final design fits in a $40 \mathrm{~cm} \times 50 \mathrm{~cm} \times 90 \mathrm{~cm}$ box and nearly lies in a $2 \mathrm{D}$ plane.

$$
\text { MIRA }
$$

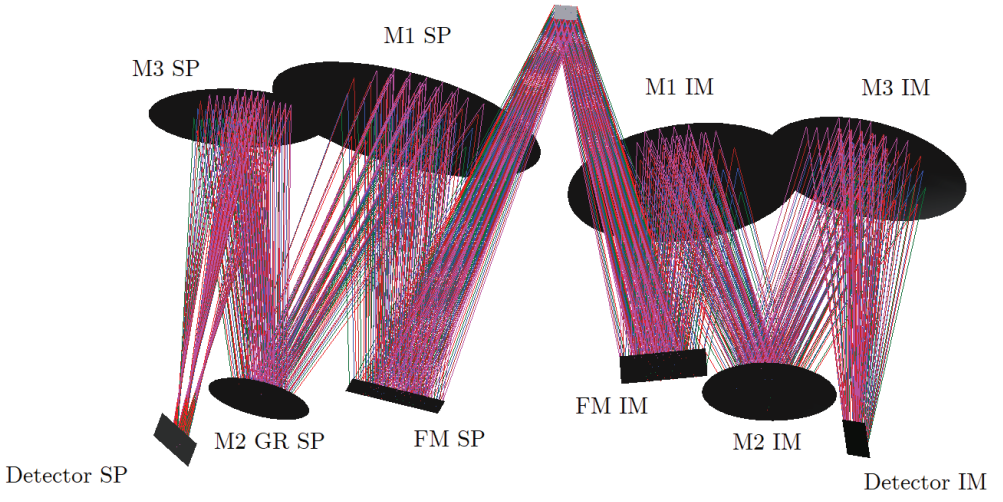

Fig. 7: Spectro-imager design for Earth observation featuring the spectrograph arm in the left-hand side of MIRA and the imaging arm on the right-hand side.

The spectro-imager optical design is summed up in table 3. 


\section{Spectro-Imager}

\begin{tabular}{ccc}
\hline Parameter & Result & Requirement \\
\cline { 1 - 2 } Mirrors & 6 aspheres $<16 \mathrm{mrad}$ & $<40 \mathrm{mrad}$ \\
\hline Mirror diameters & $<318 \mathrm{~mm}$ & \\
\cline { 1 - 2 } $\begin{array}{c}\text { Image quality after } \\
\text { tolerancing }\end{array}$ & $\begin{array}{c}11 \mathrm{\mu m} \text { for } 99 \% \text { of FOV points } \\
\text { (Monte-Carlo simulation) }\end{array}$ & $\begin{array}{c}<11 \mathrm{\mu m} \text { after tolerancing } \\
\text { procedure }\end{array}$ \\
\hline Spectral resolution & $\begin{array}{c}\text { Mean of } 1,500 \text { over } \\
{[400 \mathrm{~nm}-800 \mathrm{~nm}]}\end{array}$ & $>1,000$ \\
\hline Spatial resolution & $9 \mathrm{~m} / 2$ detector pixel & $<30 \mathrm{~m} / 2$ detector pixel \\
\hline Volume & $40 \mathrm{~cm} \mathrm{x} 50 \mathrm{~cm} \mathrm{x} 90 \mathrm{~cm}$ & \\
\hline
\end{tabular}

Table 3: Spectro-imager for Earth observation optical design results with respect to instrument specifications

The spectro-imager design for Earth observation meets all instrument requirements in terms of image quality over the $400 \mathrm{~nm}-800 \mathrm{~nm}$ wavelength range for both imager and spectrograph. A spatial resolution of $9 \mathrm{~m} / 2$ detector pixels is achieved, three times better than the initial instrument specification. The spectral resolution reaches a mean of 1,500 over the wavelength range and the final spectro-imager design fits in a $40 \mathrm{~cm}$ x $50 \mathrm{~cm}$ x $90 \mathrm{~cm}$ box.

\subsection{Conclusion: Earth observation}

The spectro-imager fits the medium resolution class of Earth observing instruments and meets all requirements set in section 2.2. For this design, the use of a MOEMS device at the intermediate focal plane of the entry telescope increases both instrument compacity and spectroscopic abilities such as simultaneous MOS and IFU. No showstopper has been highlighted for the use of MIRA in space environment and as the designed instrument has similar characteristics to instruments such as CHIME or ELOIS, MOEMS-based designs appear as strong candidates for Earth observation space missions.

\section{SPECTRO-IMAGER FOR UNIVERSE OBSERVATION}

A new MOEMS-based spectro-imager design for Universe observation is presented, achieving both high spectral and spatial resolution over the whole FOV.

\subsection{Design overview}

This instrument utilizes the same MOEMS device as the BATMAN instrument ${ }^{11}$, a DMD, on which focuses the outcoming light from the telescope. The micro-mirrors of the DMD then act as programmable slit masks, sending the corresponding FOV of the selected objects to the spectrograph, the remaining light being directed to the imager. This spectro-imager design exploits the exquisite image quality delivered by Multi Conjugate Adaptive Optics (MCAO) over a large field of view. By using several wavefront sensors and guide stars instead of one for classic adaptive optics, a 3D tomographic reconstruction of the perturbations induced by the atmosphere is obtained. These perturbations are corrected by a series of deformable mirrors, making the MCAO able to correct the whole FOV of some of the largest ground-based telescopes with homogeneous image quality. MCAO has already been demonstrated in the Near-Infrared by MAD $(\mathrm{ESO})^{18}$ and GeMs (Gemini) ${ }^{19}$. MCAO in the visible is to be demonstrated by MAVIS ${ }^{20}$ at VLT's UT4.

The concept of the spectro-imager is shown in Fig. 8. The input of this instrument is the Multi-Conjugate Adaptive Optics module (MCAO) output, corresponding to the telescope's image corrected of atmospheric perturbations. A foreoptics is then used to adapt the F-number of the outcoming beam on the DMD. A FOV as large as 30' $\mathrm{x} 30$ ', corrected by the MCAO is then re-imaged at F/12.4 onto the DMD by the fore-optics. The FOV is projected on the DMD on a total 
of 1080 x 1080 micro-mirrors. When the micro-mirrors are tilted on $\mathrm{ON}\left(+12^{\circ}\right)$ position, the light from the selected objects is directed to the spectrograph, reaching a spectral resolution of 15,000 on a single CCD. When the micro-mirrors are tilted in OFF $\left(-12^{\circ}\right)$ position, the light is directed towards the imager. A spatial resolution of 7.5 mas / detector pixel is reached over the 30 "' $\mathrm{x} 30^{\prime}$ " FOV.

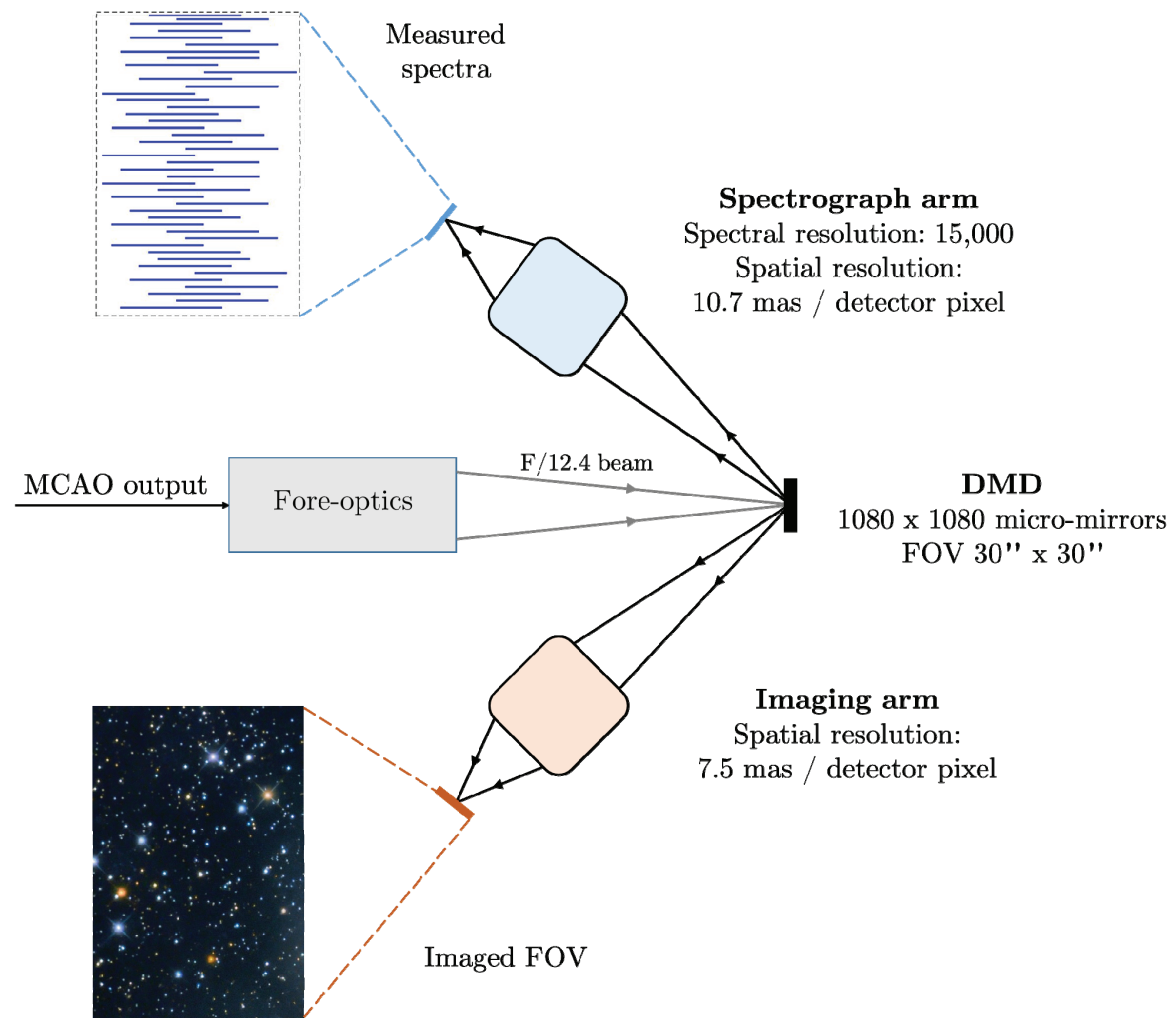

Fig. 8: Universe observation MOEMS-based spectro-imager concept: the light arrives from the left and focuses on the DMD after F-number correction by the fore-opics. The light from the selected objects in the FOV is sent to the spectrograph arm (upwards), while the remaining light is sent to the imaging arm (downwards).

\subsection{Instrument requirements}

Science cases for Universe observation are ranging from remote galaxies to the Milky Way. In order to observe intermediate to high redshift galaxies $(0.1<\mathrm{z}<5)$ with a spatial resolution of 0.025 " (200 parsecs on the object), a diffraction-limited 8-m class telescope is required. An 8.2 primary mirror diameter is chosen for the design as the actual largest ground-based non-segmented M1, corresponding to the VLT's UT primary mirror diameters and identical to the MAVIS instrument ${ }^{20}$. However, the $2 \mathrm{D} \mathrm{FOV} \mathrm{of} \mathrm{such} \mathrm{a} \mathrm{telescope} \mathrm{must} \mathrm{be} \mathrm{corrected} \mathrm{of} \mathrm{atmospheric} \mathrm{turbulence} \mathrm{to} \mathrm{reach} \mathrm{a}$ diffraction limited image quality in the telescope plane. The use of a MCAO system is therefore mandatory for a wellcorrected entry FOV for the spectro-imager; still, MCAO in the visible and over a large FOV remains a challenge and typical sizes of MCAO-corrected FOVs for 8-m class telescopes are 30" x 30"'. Typical sizes of XUV and low surface brightness galaxies are between 1-2 $\operatorname{arcmin}^{2}$. With a FOV of 30"x 30", mapping of these galaxies can be done in 4-8 observation runs, and will be even more efficient with the use of MOS and IFU over the whole FOV. A FOV of 30 ' $\mathrm{x} 30$ ', is set for the instrument.

By adjusting the focal length of both imager and spectrograph, the spatial resolution has been chosen as 0.011 " per detector pixel for the spectrograph and 0.0075 " per detector pixel for the imager, the maximum reachable spatial resolution for each instrument with respect to the chosen detectors. Moreover, a higher spatial resolution is achieved for the imager as the display of spectra in the spectrograph arm takes most of the detector area. For studying the kinematics of remote and intermediate galaxies, a velocity accuracy better than $20 \mathrm{~km} / \mathrm{s}$ is required: a spectral resolution of 15000 
has been chosen for the spectrograph. A typical slit is created by one micro-mirror, then imaged onto 2 x 2 detector pixels for a spectral resolution of 15000 . The use of different slit sizes change the spectral resolution to 8000 if the slit size is set to $2 \times 2$ micro-mirrors. These performances are still around three times better than scheduled instruments ${ }^{4}$ in terms of spectral resolution. The wavelength range of the spectro-imager has been extended from the visible to the near infrared [370 nm $-950 \mathrm{~nm}$ ] and is divided into 4 sub-bands to achieve a spectral resolution of 15000 over the whole wavelength range while using a single CCD. The sub-bands are as follows: [370 nm $-470 \mathrm{~nm}],[470 \mathrm{~nm}-590 \mathrm{~nm}]$, $[590 \mathrm{~nm}-750 \mathrm{~nm}]$ and $[750 \mathrm{~nm}-950 \mathrm{~nm}]$, chosen as to achieve the best homogeneity in terms of spectral resolution over the whole wavelength range. The sub-bands are analyzed successively by using a grating wheel while the collimator and camera are the same for all sub-bands.

The square 30"'x 30" FOV is finally projected on the maximum available square DMD surface i.e. $1080 \mathrm{x} 1080$ mirrors, for an equivalent projected FOV of 27.7 mas per micro-mirror. A F-number of 12.4 for the incoming light onto the DMD is chosen for imaging the $30^{\prime}$ x $30^{\prime}$ 'FOV on $14 \mathrm{~mm} \times 14 \mathrm{~mm}$ DMD plane. The light incomes at normal incidence on the DMD and outcomes at $24^{\circ}$ due to the micro-mirrors' $12^{\circ}$ tilt. The light goes through the imager or the spectrograph and focuses on the detector planes. The chosen detector for the imager is the CCD e2v CCD250, featuring $10 \mu \mathrm{m}^{2}$ pixels in 4096 x 4004 array. For the spectrograph, the detector is chosen as the largest available for astronomical spectroscopy, featuring $9216 \times 9232$ pixels being each $10 \mu \mathrm{m}^{2}$.

The instrument specifications are summed up in table 4.

\section{Instrument specifications}

\begin{tabular}{|c|c|}
\hline Primary mirror diameter & $8.2 \mathrm{~m}$ \\
\hline Field of view & $30 \operatorname{arcsec} \times 30 \operatorname{arcsec}$ \\
\hline Focal ratio at $\mathrm{AOM}$ output & $\mathrm{F} / 35$ \\
\hline Wavelength range & $370-950 \mathrm{~nm}$ \\
\hline Beams on DMD & $\begin{array}{c}\text { Incoming light at normal incidence } \\
\text { Outcoming light at } 24^{\circ} \\
\text { DMD orientation at } 45^{\circ}\end{array}$ \\
\hline Focal ratio on DMD & $\begin{array}{l}\text { F/12.4 on DMD ( } 1080 \times 1080 \text { micro- } \\
\text { mirrors) } \\
\text { Plate scale: } 27.7 \text { mas per micro-mirror } \\
\text { Micro-mirror pitch: } 13.68 \mu \mathrm{m}\end{array}$ \\
\hline Imaging arm spatial resolution & $\begin{array}{c}7.5 \text { mas / detector pixel } \\
\text { Detector: CCD e2v CCD250 } \\
4 \mathrm{k} \times 4 \mathrm{k} \text { pixels, pixel size: } 10 \mu \mathrm{m}\end{array}$ \\
\hline Spectral resolution & $\begin{array}{l}\mathrm{R}=15,000 \text { for the full wavelength and the } \\
\text { full FOV } \\
1 \text { micro-mirror on } 2 \times 2 \text { detector pixels } \\
\text { Spatial resolution: } 10.7 \text { mas / detector pixel } \\
\text { Detector: CCD e2v CCD250 } \\
\text { 9k x } 9 \mathrm{k} \text { pixels, pixel size: } 10 \mu \mathrm{m}\end{array}$ \\
\hline $\begin{array}{l}\text { Spectral bands (use of a grating wheel } \\
\text { to access the four bands) }\end{array}$ & $\begin{array}{l}\text { Four spectral bands: } \\
\text { Band 1: } 370-470 \mathrm{~nm} \\
\text { Band 2: } 470-590 \mathrm{~nm} \\
\text { Band 3: } 590-750 \mathrm{~nm} \\
\text { Band 4: } 750-950 \mathrm{~nm}\end{array}$ \\
\hline
\end{tabular}

Table 4 - Spectro-imager instrument specifications for Universe Observation

\subsection{Observational modes}

Individual control of each micro-mirror enables optimized observation strategies: the size and shape of the spectrograph's entrance slits can be chosen over the whole FOV, adding the possibility to vary the spatial element resolution and spectral resolution for the MOS. Both slit shape and size can be reconfigured in real time. IFU can be 
done simultaneously to MOS in the whole FOV, minimizing the number of observation runs needed by sequentially scanning a long slit along the object of interest. Due to the fast switching of the micro-mirrors, imagery is quasisimultaneously available with spectroscopy; individual control of each micro-mirror also guarantees an independent access to each object of the FOV, in both spatial and spectral domains. New observation strategies are available to optimize the recorded data over the FOV.

\subsection{Imager}

For the imager, the main goal was to design a simple and compact optical system to achieve high spatial resolution imagery with a projection of the FOV equivalent to 7.5 mas per detector pixel. This way, the intermediate image focused on the DMD is re-imaged on the $40 \mathrm{~mm} \times 40 \mathrm{~mm}$ CCD with a magnification of 2.72. The DMD is located at the upper right of the layout of the imager in Fig. 9 and is followed by a freeform concave-convex two-mirrors configuration with low local slopes.

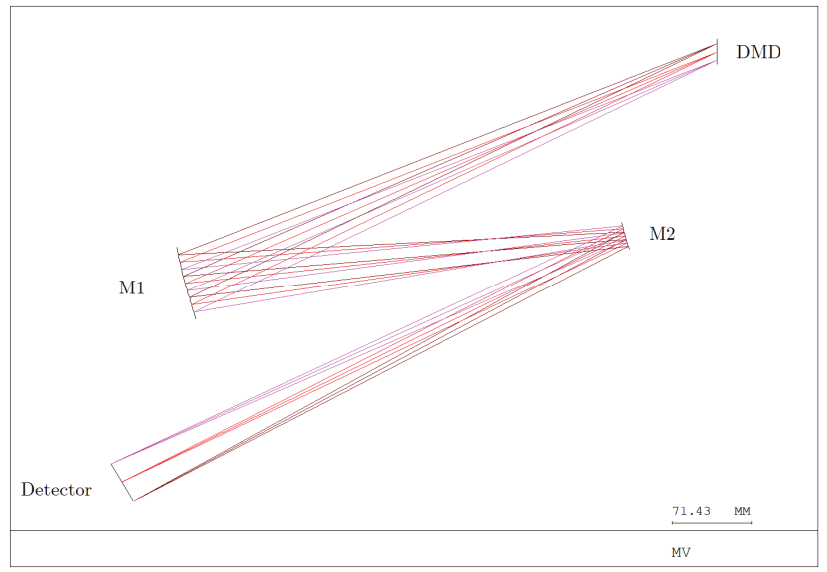

Fig. 9: 2D layout of the imaging arm of the Universe observation spectro-imager.

From top to bottom, Fig. 9 features: the DMD, a concave M1 mirror of $63 \mathrm{~mm}$ diameter (left), a convex M2 mirror of $23 \mathrm{~mm}$ diameter (right) and the detector. The imager fits in a $60 \mathrm{~cm} \mathrm{x} 45 \mathrm{~cm}$ box. To visualize the image quality over the whole FOV, spot diagrams at the center and edges of the FOV and a color contour plot of the spot diagrams values over the whole FOV projected on the detector are represented in Fig. 10.

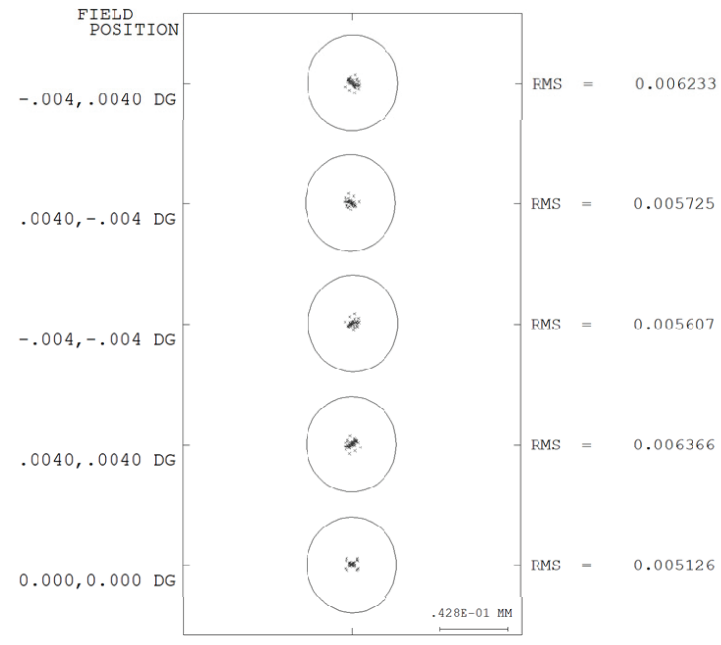

(a)

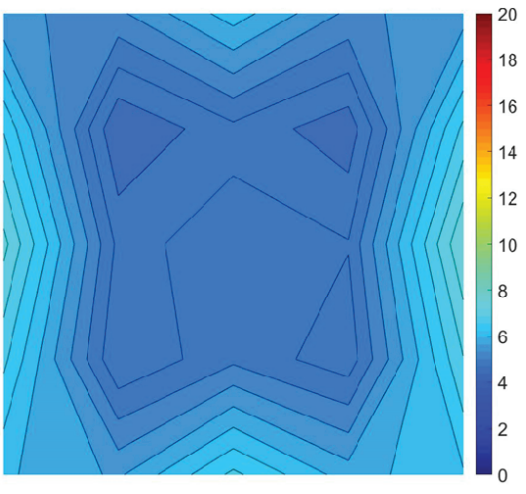

(b)

Fig. 10: Imager for Universe observation nominal image quality a) Spot diagrams $(\mu \mathrm{m})$ at the center and edges of the FOV overlaid on top of the Airy disk representing the diffraction limit; b) Spot diagram cartography $(\mu \mathrm{m})$ for 25 points in the 30"x30" FOV. 
The imager is diffraction limited, as shown in Fig. 10a. The maximum of the scale used in Fig. 10b corresponds to the spot diagram instrument specification, $20 \mu \mathrm{m}$. The mean image quality is equal to $5.8 \mu \mathrm{m}$ for a standard deviation of $0.9 \mu \mathrm{m}$, confirming the homogeneity of the imager. The best image quality appears for intermediate FOV points and reaches spot diagrams as low as $4.4 \mu \mathrm{m}$.

\subsection{Tolerancing}

Tolerancing consists in the simulation and analysis of all possible error sources that will lead the final image quality of the system to degrade after installation. These optical errors can be divided in two main sources: manufacturing errors on the optics and actual alignment procedure errors, leading to small mispositioning of the optics which result in residual tilt, decenter and defocus errors and degrade the nominal image quality of the system once it is aligned. By decomposing the sources of errors leading to degradation in performances, we are able to simulate the image quality of any optical system once it has been aligned. The tolerancing procedure is divided in 4 steps as indicated in Fig. 11.

The first step emerges from the resulting errors of the tools used to polish the optical surface. This step thus consists in simulating the errors due to the manufacturing of the optics; however, such a simulation implies a relative understanding of surface topography characterization: surface characterization is to be explained and transitioned in simulation of the manufacturing errors in Matlab and CodeV.

The second step consists in the simulation of the errors coming from the initial alignment of the optics. When aligned at first, the optical surfaces and the detector will be misaligned due to the tool precision and the procedure followed to align the system. This translates in additional decenters, tilt and defocus errors for each optical surface and results in increased aberrations in the detector plane. To be able to reproduce these errors, the same mis-alignments are applied on the nominal CodeV file studied for a large number of systems.

The third step of the tolerancing process then consists in the realignment of the optics to obtain the optimal PSF over the whole field of view, and translates in Code $\mathrm{V}$ as a re-optimization of the system, using the focus, decenter and tilt of the optics as variables.

The last step of the tolerancing consists in taking into account the minimal compensator step of the opto-mechanical mounts on which is positioned each optical surface. While aligning the real system on bench, reaching the same precision as Code V's optimizer is impossible because of the minimal reachable steps on each mount. Compensators are linked to the size of the shims used to the last adjustment of the optics, which is usually around $10 \mu \mathrm{m}$ in decenter; theoretically, a better precision could be achieved but would imply greatly increased costs. These values are then applied on the optical system after the re-optimization and the final system after tolerancing is obtained.

The tolerancing procedure is then repeated a large number of times to obtain a reliable statistical response of the optical system to tolerancing; for that, a Monte Carlo simulation is used, where 250 systems with random initial tilts and decenters are considered. The four steps of tolerancing are applied successively to any considered optical system and the chosen criterion for the image quality is the RMS spot diagram. If the post-tolerancing instrument shows degradations in image quality exceeding two detector pixels, it is re-aligned - if the image quality is below two detector pixels, the final instrument is obtained.

Polishing leads to errors covering a wide frequency range for optical surfaces i.e. mirror or lenses. The contribution of different wavevectors is usually divided in three parts: low, mid and high spatial frequencies. The resulting Power Spectral Density (PSD) of the surface is not perfectly logarithmic but covered by noise and peaks corresponding to the ripple frequency of the polishing instrument. The term "mid-spatial frequency" is in fact ambiguous and its definition depends on the other frequency ranges. Low frequency errors transfer the energy for the core of the PSF to the first diffraction rings, without broadening the core of the PSF. These spatial frequencies are perfectly described by the Zernike polynomials as low spatial frequencies are equivalent to the optical aberrations of the system. Low spatial frequencies, also called "figure" are corrected by the optimization and minimization of the aberrations in the detector plane. High frequency errors, also called "roughness", scatter the energy out of the PSF core to a wide halo again without broadening the core of the PSF. Mid-spatial frequencies (MSF) however produce small angle scatter which significantly degrades the core the PSF, resulting in decreased image quality. MSF cannot therefore be treated in the same aspect as aberration theory or a global loss in transmission and must be simulated for further evaluation. 䙵he MSF range can be defined as beginning with the highest terms in the Zernike expansion, which is equivalent to spatial frequencies around or about 5-10 cycles over the aperture of the optic. MSF can be modeled by using sinusoidal waves with different frequencies. A randomized period between 5 and 10 times smaller than each mirror's aperture is chosen. The amplitude 
of the sinusoids is dependent on the tool used for polishing the surface: typical polishing methods are precise to $\lambda / 10$ in $\mathrm{PV}$, which then corresponds to $60 \mathrm{~nm}$ PV. An average PV value of $70 \mathrm{~nm}$ for an average RMS of $12 \mathrm{~nm}$ is chosen, slightly worse than typical manufacturing capabilities.

\section{TOLERANCING}

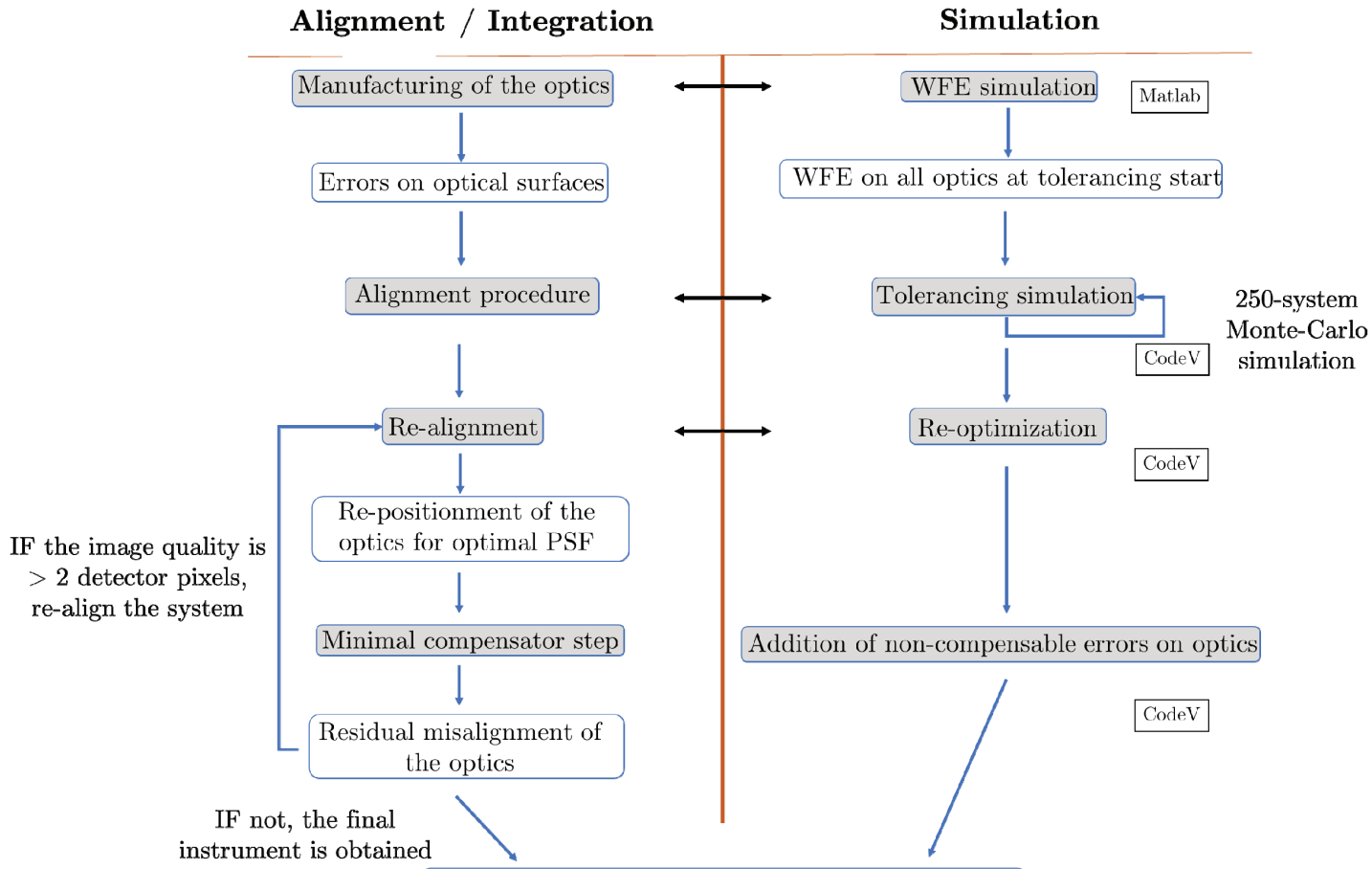

Final instrument after tolerancing

Fig. 11: Tolerancing principle: equivalence between the alignment (left) and the Matlab and CodeV simulation codes (right).

Manufacturing errors have been simulated under Matlab and added on the three mirrors of the imager. The tolerancing process for the imager includes simulation of manufacturing errors on the mirrors, alignment errors and residual non-compensable errors. After tolerancing and over the FOV, the global loss of image quality is around $5.6 \mu \mathrm{m}$, for a mean spot diagram value after tolerancing of $11.4 \mu \mathrm{m}$. The number of points in the FOV that stay below $20 \mu \mathrm{m}$ have been counted, for a total of $6196 / 6250$ in the 250 systems simulation, which corresponds to $0.86 \%$ of the spot diagrams. On average, $99.14 \%$ of the spot diagrams after tolerancing are within instrument specifications. The imager therefore reaches the instrument specifications in both terms of optical surfaces and image quality after tolerancing.

\subsection{Spectrograph}

The main requirements for the spectrograph are high spectral resolution (mean of 15,000) over the whole FOV and the whole wavelength range, and a spatial resolution equivalent to 10.7 mas / detector pixel. Moreover, all spectra must be displayed on a single $9 \mathrm{k} \times$ 9 $\mathrm{k}$ CCD detector, and, the wavelength range being divided in four bands, the collimator and the camera must be the same for all wavelength bands. To be able to image the four bands on a single CCD, a grating wheel is used, on which the grating, referred to as M2 GR, is placed. Finally, the number of mirrors has to be minimized to have the highest instrument throughput and leads to the use of freeform optics.

The final spectrograph design is a three freeform mirror instrument (Fig. 12): one for the collimator, one on which is located the grating and one mirror for the camera. By placing 4 gratings in a grating wheel at the M2 GR location, switching between each wavelength band is possible. M1 and M3 remain identical for the whole wavelength range and all wavelength bands are displayed on the same detector, with all spectra for the same point of the projected FOV nearly overlapping. 


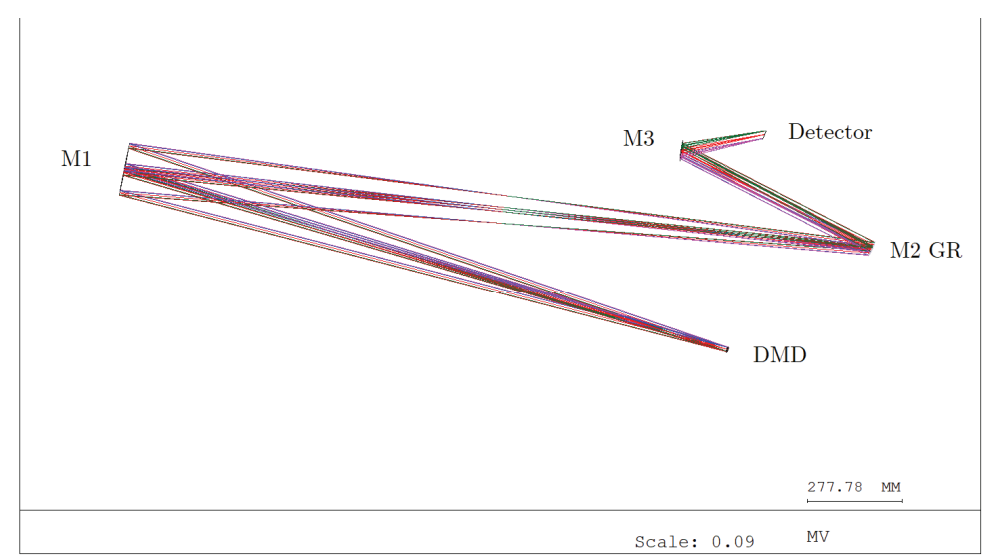

Fig. 12: 2D layout of the spectrograph arm of the Universe observation spectro-imager.

All mirrors are freeform but all have rather low local slopes $(<22 \mathrm{mrad})$. Four reflective gratings are used, and while they have the same shape, each has a different grating line density in order to reach a median spectral resolution of 15000 per wavelength band. Realization of a grating on a freeform surface is still a challenge; a joint program between LAM and CSEM (Switzerland) is currently developing an innovative technology to be able to realize these gratings. ${ }^{21,22}$

Figure 13a features the spot diagrams at the center of the FOV for the center and extreme wavelengths of each spectral band. the spectrograph is nearly diffraction limited over the whole wavelength range. A cartography of the whole FOV for all center and extreme wavelengths of every spectral band has been completed to analyze the spectrograph's spot diagram homogeneity (Fig. 13b).

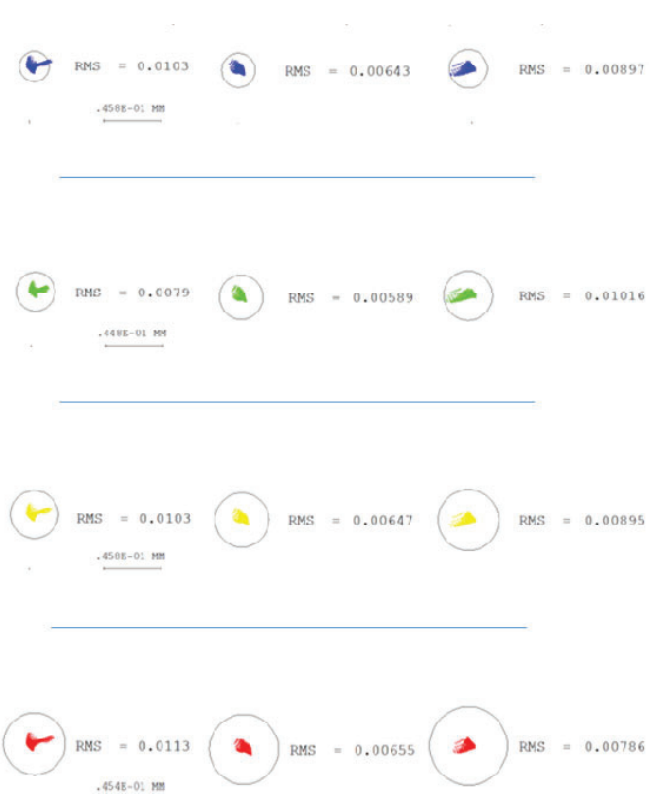

(a)
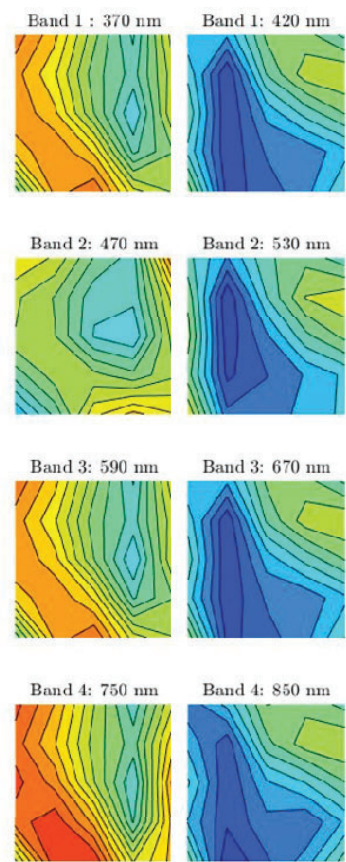

(b)
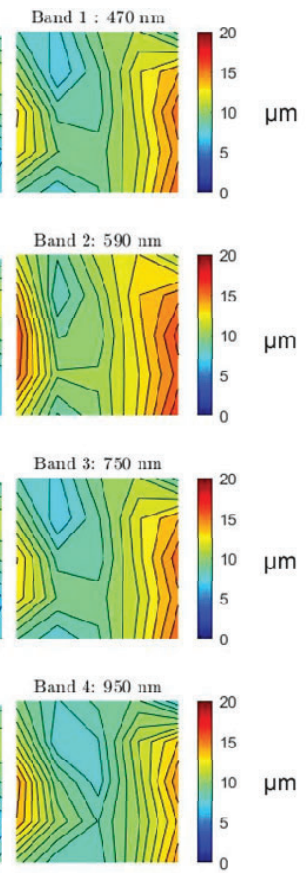

Fig. 13: Spectrograph for Universe observation nominal image quality a) Spot diagrams $(\mu \mathrm{m})$ at the center of the FOV for the extreme and center wavelengths of each wavelength band overlaid on top of the Airy disk representing the diffraction limit; b) spot diagram cartography $(\mu \mathrm{m})$ for the extreme and center wavelengths of each wavelength band. 
The cartography has been divided per wavelength per spectral band, and the colorbar used in Fig. 13b is set to have a maximum of $20 \mu \mathrm{m}$ which corresponds to the instrument specification. Figure 13b shows the spot diagrams at the center of the bands to be better than for the extreme wavelengths: the four centers of the bands have a mean spot value of $7.4 \mu \mathrm{m}$, while extreme wavelengths have a mean spot value of $11.6 \mu \mathrm{m}$. Spot diagrams are better at center wavelengths and remains under $17 \mu \mathrm{m}$ for the whole FOV.

The tolerancing procedure takes into account manufacturing errors on the mirrors, alignment errors and non-compensable errors with the addition of a non-compensable tilt error due to the grating position error introduced by the use of a grating wheel. Over 250 tolerancing runs, The average spot diagrams of all wavelength are below $17.2 \mu \mathrm{m}$, and $99.1 \%$ of the spot diagrams are below $20 \mu \mathrm{m}$. The analysis of the final wave front error after accounting for manufacturing and alignment errors has proven the spectrograph to meet the instrument requirement in terms of image quality.

The projected FOVs and the spectra for the first wavelength band are shown in Fig. 14. Due to the spectra length of $70 \mathrm{~mm}$, the only solution to fit all spectra in a single CCD is to tilt the CCD with respect to the spectra, as shown in Fig. 14. Figure 14a then shows all spectra for the first band to fit in the single CCD - and all four wavelength bands are nearly superposed to each other. The projected FOVs for the center and extreme wavelengths of band 1, respectively plotted in black and blue \& red in Fig. 14b, then appears as diagonally tilted with respect to the CCD. All spectra tightly fit in a single detector.

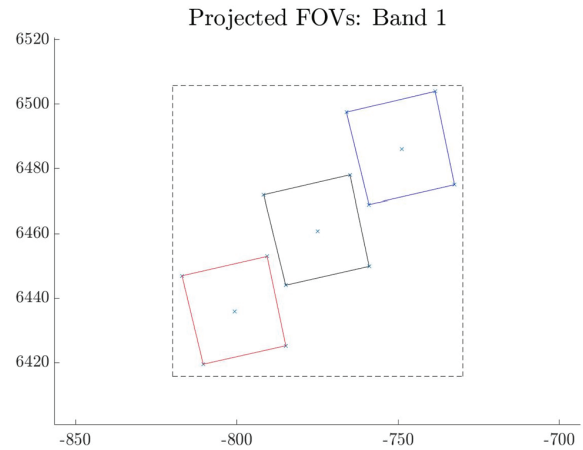

(a)

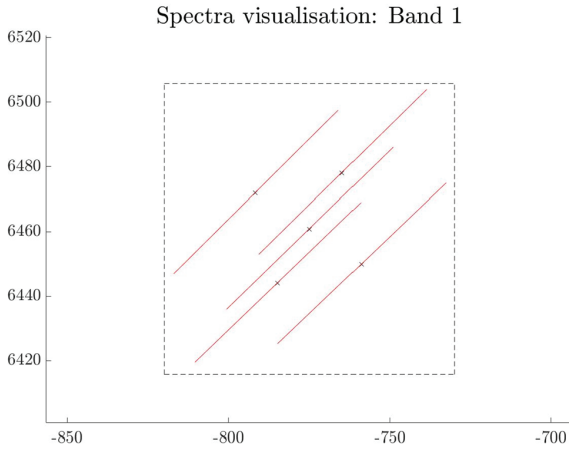

(b)

Fig. 14: Universe observation spectrograph detector plane view: a) Projected FOV for extreme and central wavelength of wavelength band 1 ( $370-470 \mathrm{~nm}$ overlaid on top of a box representing the detector); b) Spectra visualization for the first wavelength band at center wavelength $(420 \mathrm{~nm})$ and for center and four edges of the 30"x30" FOV.

The computation of the spectral resolution has been done over the four wavelength bands and the spectral resolutions for the extreme and center wavelengths for each wavelength band are shown in table 5. A mean spectral resolution of around 15000 has been obtained for each wavelength band. The starting spectral resolution for each band is around 13000 and the spectral resolution of 15000 is reached near the center of each spectral band. A spectral resolution as high as 17200 is achieved at the end of each spectral band, for an average of 16670 .

\begin{tabular}{|c|c|c|c|c|}
\hline & & $B$ and 1 & & B and width (nm ) \\
\hline W avelength (nm) & 370 & 420 & 470 & 100 \\
\hline Spectral resolution & 12950 & 14700 & 16450 & \\
\hline & & $\mathrm{B}$ and 2 & & B and width (nm ) \\
\hline W avelength (nm) & 470 & 530 & 590 & 120 \\
\hline Spectral resolution & 13708 & 15458 & 17208 & \\
\hline & & $\mathrm{B}$ and 3 & & B and width (nm) \\
\hline W avelength $(\mathrm{nm})$ & 590 & 670 & 750 & 160 \\
\hline Spectral resolution & 12906 & 14656 & 16406 & \\
\hline & & B and 4 & & $\mathrm{~B}$ and $\mathrm{w}$ idth (nm) \\
\hline W avelength $(\mathrm{nm})$ & 750 & 850 & 950 & 200 \\
\hline Spectral resolution & 13125 & 14875 & 16625 & \\
\hline
\end{tabular}

Table 5: Universe observation spectrograph spectral resolution over the four wavelength bands $(370-950 \mathrm{~nm})$ 


\subsection{Spectro-imager}

By assembling the two designs on each side of the DMD, the final layout of the spectro-imager design for Universe observation is obtained and presented in Fig. 15.

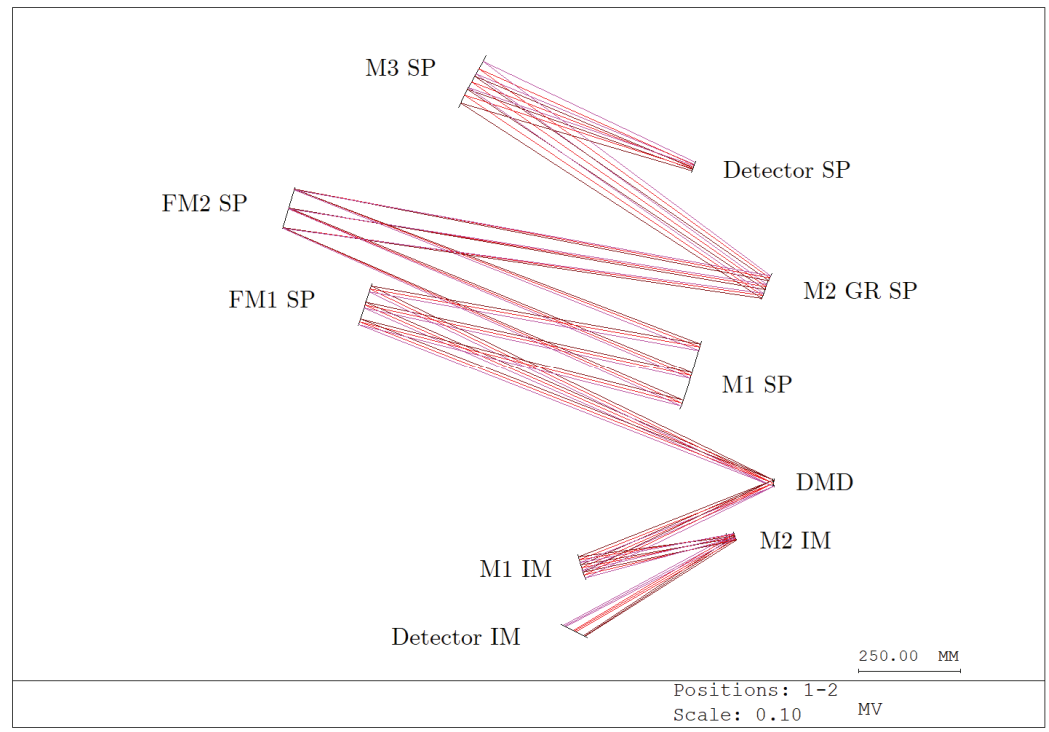

Fig. 15: Spectro-imager design for Universe observation featuring the spectrograph arm in the upper side of the DMD and the imaging arm on the lower side.

Figure 15 features both imager and spectrograph with two additional fold mirrors in the spectrograph arm to increase instrument compacity. The final spectro-imager fits in a $1.4 \mathrm{~m} \mathrm{x} 1.4 \mathrm{~m}$ box and the off plane of the optical design has been minimized to $5 \mathrm{~cm}$. The use of freeform surfaces allows for a two-mirror imager and a two mirror and 1 grating spectrograph, resulting in a simple spectro-imager. The overall design is then featuring 4 freeform mirrors and 4 freeform gratings. The spectro-imager design is summed up in table 6.

\section{Spectro-Imager}

\begin{tabular}{|c|c|c|}
\hline Parameter & Result & Requirement \\
\hline Mirrors & 4 freeforms mirrors $<22 \mathrm{mrad}$ & \multirow{3}{*}{$<40 \mathrm{mrad}$} \\
\hline Gratings & 4 freeform gratings $<3 \mathrm{mrad}$ & \\
\hline Mirror diameters & $<180 \mathrm{~mm}$ & \\
\hline $\begin{array}{l}\text { Image quality after } \\
\text { tolerancing }\end{array}$ & $\begin{array}{c}<20 \mu \mathrm{m} \text { for } 99 \% \text { of FOV points } \\
\text { (Monte-Carlo simulation) }\end{array}$ & $\begin{array}{c}<20 \mu \mathrm{m} \text { after tolerancing } \\
\text { procedure }\end{array}$ \\
\hline Spectral resolution & $\begin{array}{l}\text { Mean of } 15000 \text { over } \\
{[370 \mathrm{~nm}-950 \mathrm{~nm}]}\end{array}$ & 15000 \\
\hline Spatial resolution & 7.5 mas / detector pixel & 7.5 mas / detector pixel \\
\hline Volume & $1.4 \mathrm{~m} \mathrm{x} 1.4 \mathrm{~m}$ & \\
\hline
\end{tabular}

Table 6: Spectro-imager for Universe observation optical design results with respect to instrument specifications 
The spectro-imager meets all instrument specifications, achieving high spatial and spectral resolution over the [370 nm - $950 \mathrm{~nm}]$ wavelength range for both imager and spectrograph. All instrument specifications are met for both arms, the image quality after tolerancing being within two detector pixels.

\subsection{Conclusion: Universe observation}

A MOEMS-based spectro-imager for 8-meter class telescopes with MCAO system has been successfully designed. The use of a MOEMS device allows for increased instrument compacity and multi-object spectroscopy over the 30 " x 30" FOV. New observational modes are also possible with the use of the DMD at the focal plane of the telescope, increasing observation efficiency and combining MOS and IFU in the whole FOV. Both imager and spectrograph are near diffraction-limited, achieving a spatial resolution of 7.5 mas and a mean spectral resolution of 15000 . By using a grating wheel and a single mirror as a camera for the $[370 \mathrm{~nm}-950 \mathrm{~nm}$ ] wavelength range, the optical design is simple in addition of having unique performances with respect to present instruments behind the 8-meter class telescopes. This spectro-imager design fully proves both the validity of MOEMS for competitive Universe observation instruments and the potential of freeform optics to simplify optical designs while increasing image quality.

\section{CONCLUSION}

Two new MOEMS-based spectro-imagers suitable for Earth and Universe observation with unique performances and observational modes have been successfully designed. The use of MOEMS proved for increased instrument performances such as increased compacity or near-simultaneous imagery and spectroscopy with MOS and IFU available at the same time; the two MOEMS devices, MIRA and DMD, are perfect components for slit spectroscopy over a 2D FOV for Earth and Universe observation.

The spectro-imager for Earth observation reaches medium spatial and spectral resolutions and is oriented towards Earth surface characterization from space for agriculture, food security and management of natural resources. This design illustrates the spectroscopic abilities of MOEMS-based instruments for space missions and a resulting increase in instrument compacity for hyperspectral imaging. Imager and spectrograph fit in a $40 \mathrm{~cm} \mathrm{x} 50 \mathrm{~cm} \mathrm{x} 90 \mathrm{~cm}$ box and reach medium spectral and spatial resolutions. The spectro-imager fits the specifications set for this class of space-borne instruments. With characteristics close to recent instruments such as CHIME or ELOIS, MOEMS-based hyperspectral imaging seems like a great candidate for Earth observation.

The spectro-imager for Universe observation achieves high spatial and spectral resolution and is designed to lie behind an 8-meter class telescope and a MCAO. It consists in a complex freeform optical design, reaching high spatial and spectral resolutions using a minimal number of optical surfaces -4 freeform mirrors and 4 freeform gratings with low local slopes, the 4 grating differing only by the grating line densities on their surfaces. The use of a grating wheel was key to divide the wavelength range in spectral bands, thus featuring a single mirror for the collimator, for the camera and also a single detector. Numerous science cases in Universe observation benefit from a mean spectral resolution of 15,000. This instrument demonstrates the reachable performances for MOEMS-based spectroscopy for Universe observation.

For Universe and Earth observation, individual control of each micro-mirror enables optimized observation strategies: the size and shape of the spectrograph's entrance slits can be chosen over the whole FOV, adding the possibility to vary the spatial element resolution and spectral resolution to MOS. IFU can be done simultaneously to MOS in the whole FOV, minimizing the number of observation runs needed.

MOEMs-based spectro-imagers are suitable for Earth and Universe observation, with unique performances both in spatial and spectral domains, allowing new observational modes.

\section{ACKNOWLEDGEMENT}

The authors would like to thank the French Space Agency (Centre National d'Etudes Spatiales, CNES) for partly funding this work. 


\section{REFERENCES}

[1] Paz, A.G., Madore, B.F., Boissier, S., Swaters, R., Popescu, C.C., Tuffs, R.J., Sheth, K., Kennicutt, R.C., Bianchi, L., Thilker, D., Observatories, D.C., Maryland, U.O., Mpik, Germany., CaltechSSC, Observatories, S.Y., Jhu, \& Caltech, "Discovery of an Extended Ultraviolet Disk in the Nearby Galaxy NGC 4625", The Astrophysical Journal, 627, 29. (2005)

[2] O'neil, K., Bothun, G.D., \& Schombert, J.M., "The Effects of Starburst Activity on Low Surface Brightness Disk Galaxies", The Astronomical Journal, 116, 2776-2792. (1998)

[3] Contini, T., Epinat, B., Bouché, N., Brinchmann, J., Boogaard, L.A., Ventou, E., Bacon, R., Richard, J., Weilbacher, P.M., Wisotzki, L., Krajnovi'c, D., Vielfaure, J., Emsellem, E., Finley, H., Inami, H., Schaye, J., Swinbank, M., Guérou, A., Martinsson, T.P., Michel-Dansac, L., Schroetter, I., Shirazi, M., \& Soucail, G. "Deep MUSE observations in the HDFS Morpho-kinematics of distant star-forming galaxies down to 108M", Astronomy and Astrophysics, 591. (2016)

[4] Henault, F. "MUSE optomechanical design and performance", Proc. SPIE 5492, Ground-based Instrumentation for Astronomy, (2004)

[5] Hajime Sugai et al, "Prime Focus Spectrograph for the Subaru telescope: massively multiplexed optical and near-infrared fiber spectrograph," J. Astron. Telesc. Instrum. Syst. 1(3) 035001 (2015)

[6] Zamkotsian F., Dohlen K., Burgarella D., Buat V., "Aspects of MMA for MOS: optical modeling and surface characterization, spectrograph optical design", in Proceedings of the NASA conference on "NGST Science and Technology Exposition", ASP Conf. Ser. 207, 218-224, Hyannis, USA, (1999)

[7] Robberto M., Cimatti A., Jacobsen A., Zamkotsian F., Zerbi F. M., "Applications of DMDs for Astrophysical Research", in Proceedings of the SPIE conference on MOEMS 2009, Proc. SPIE 7210, San Jose, USA, (2009)

[8] Li, M. J.; Brown, A. D.; Kutyrev A. S.; Moseley H. S.; Mikula V., " JWST microshutter array system and beyond", Proc. SPIE 7594, San Francisco, USA (2010)

[9] Zamkotsian F., Lanzoni P., Grassi E., Barette R., Fabron C., Tangen K., Valenziano L., Marchand L., Duvet L. "Successful evaluation for space applications of the 2048x1080 DMD," in Proceedings of the SPIE conference on MOEMS 2011, Proc. SPIE 7932, San Francisco, USA (2011)

[10] Canonica M., Zamkotsian F., Lanzoni P., Noell W., de Rooij N., "The two-dimensional array of 2048 tilting micromirrors for astronomical spectroscopy," Journal of Micromechanics and Microengineering, 23 055009, (2013)

[11] Zamkotsian F. , Lanzoni P. , Tchoubaklian N. , Ramarijaona H. , Moschetti M., Riva M. , Jaquet M. , Spano P. , Bon W. , Vachey M. et al. , "BATMAN @ TNG: instrument integration and performance", Proc. SPIE 10702, Ground-based and Airborne Instrumentation for Astronomy VII, 107025P (2018)

[12] Rast, M. et al, Earth and Mission Science Division, ESA: "Copernicus Hyperspectral Imaging Mission for the Environment - Mission Requirements Document", Reference "ESA-EOPSM-CHIM-MRD-3216" (2018)

[13] De Clercq, C. \& Moreau, V. \& Jamoye, J.-F. \& Zuccaro M., Alessandro \& Gloesener, P. "ELOIS: an innovative spectrometer design using a free-form grating", Proc. SPIE 9626, SPIE Optical Systems Design. (2015)

[14] Popescu ,A. F. ; Paulsen, T. ; Ratier, G. ; Uguen, G. ; Asseman, I. ; Wilson, R. B. ; Mau, K.-D. : "Global Ozone Monitoring by Occultation of Stars (GOMOS) instrument on ENVISAT: requirements, design, and development status"; Proceedings Volume 2957, Advanced and Next-Generation Satellites II; (1997)

[15] Lamard, J.-L. \& Valentini, D. \& Laherrere, J.-M. \& Gaudin-Delrieu, C. \& Renard, C. \& Tournier, T., "Design of the high resolution optical instrument for the Pleiades HR Earth observation satellites", (2017)

[16] Courtois, M. \& Traizet, M.: "The SPOT satellites: From SPOT 1 to SPOT 4", Geocarto International, 1:3, 4-14 (1986)

[17] Canonica M., Zamkotsian F., Lanzoni P., Noell W., and Rooij N. "Mems-based programmable reflective slit mask for multiobject spectroscopy", Proc. SPIE, volume 7930, page79300N, (2011)

[18] Liuzzo, E., Falomo, R., Paiano, S., Treves, A.L., Uslenghi, M., Arcidiacono, C., Baruffolo, A., Diolaiti, E., Farinato, J., Lombini, M., Moretti, A., Ragazzoni, R., Brast, R., Donaldson, R., Kolb, J., Marchetti, E., \& Tordo, S. "MAD Adaptive Optics imaging of high luminosity quasars: a pilot project", The Astronomical Journal, 152, 38. (2016)

[19] Rigaut, F., Neichel, B., Boccas, M., d'Orgeville, C., Vidal, F., Dam, M.A., Arriagada, G., Fesquet, V., Galvez, R.L., Gausachs, G., Cavedoni, C., Ebbers, A.W., Karewicz, S., James, E., Luhrs, J., Montes, V.G., Pérez, G.R., Rambold, W.N., Rojas, R., Walker, S., Bec, M., Trancho, G., Sheehan, M.S., Irarrazaval, B.A., Boyer, C., Ellerbroek, B.L., Flicker, R.C., Gratadour, D., Garcia-Rissmann, A., \& Daruich, F. "Gemini multiconjugate adaptive optics system review - I. Design, trade-offs and integration", Monthly Notices of the Royal Astronomical Society, 437, 2361-2375. (2014)

[20] ESO, “About MAVIS”, http://mavis-ao.org/mavis/, , (2020)

[21] Zamkotsian, F., Zhurminsky, I., Lanzoni, P., Tchoubaklian, N., Schneider, C., Fricke, S., Schnieper, M., Lütolf, F., Luitot, C., \& Costes, V. "Convex blazed gratings for high throughput spectrographs in space missions", Proc. SPIE 11180, International Conference on Space Optics - ICSO 2018, 1118051. (2019)

[22] Zamkotsian, F., Zhurminsky, I., Lanzoni, P., Tchoubaklian, N., Lütolf, F., Schnieper, M., Schneider, C., Fricke, S., Fouchier, M., Zerrad, M., Amra, C., Costes, V., \& Loesel, J., "Convex blazed gratings for high throughput spectrographs in space missions", this conference, International Conference on Space Optics - ICSO 2020, (2021) 